\title{
Nontraumatic Nonaneurysmal Subarachnoid Hemorrhage: Risk Factors, Complications, and Clinical Outcomes
}

\author{
Ehsan Alimohammadi ${ }^{1}$ Paniz Ahadi ${ }^{2}$ Ali Karbasforoushan ${ }^{3}$ Shamsoddin Rahmani ${ }^{1}$ \\ Seyed Reza Bagheri ${ }^{1} \quad$ Alireza Abdi ${ }^{4}$
}

${ }^{1}$ Department of Neurosurgery, Kermanshah University of Medical

Sciences, Imam Reza Hospital, Kermanshah, Iran

${ }^{2}$ Clinical Research Development Center of Taleghani and Imam Ali Hospital, Kermanshah University of Medical Sciences, Kermanshah, Iran

${ }^{3}$ Department of Anesthesiology, Kermanshah University of Medical Sciences, Imam Reza Hospital, Kermanshah, Iran

${ }^{4}$ Nursing and Midwifery School, Kermanshah University of Medical Sciences, Imam Reza Hospital, Kermanshah, Iran

\author{
Address for correspondence Ehsan Alimohammadi, MD, \\ Department of Neurosurgery, Kermanshah University of Medical \\ Sciences, Imam Reza Hospital, Kermanshah, Iran \\ (e-mail: Hafez125@gmail.com).
}

Indian J Neurosurg 2021;1:37-41.

\begin{abstract}
Keywords

- nontraumatic nonaneurysmal subarachnoid hemorrhage

- digital subtraction angiography

- perimesencephalic

- nonperimesencephalic

- Glasgow outcome scale

Background The present study aimed to investigate the risk factors, complications, and clinical outcomes of patients with nontraumatic, nonaneurysmal subarachnoid hemorrhage (NNSAH).

Methods We retrospectively evaluated 78 consecutive patients with NNSAH admitted to our center between April 2009 and April 2019. Patients were divided into two groups based on the distribution of blood in the CT scan, perimesencephalic subarachnoid hemorrhage (PM-SAH) and nonperimesencephalic subarachnoid hemorrhage (nPM-SAH) groups. The outcome was assessed according to the Glasgow outcome scale (GOS). The demographic data and clinical records including age, sex, smoking history, hypertension, diabetes, history of anticoagulant medication, Glasgow coma score (GCS), Hunt-Hess (HH) grades, and in-hospital complications and clinical outcomes were retrospectively reviewed and compared between the two groups.

Results There were 45 patients $(57.69 \%)$ in the PM-SAH group and 33 cases $(42.30 \%)$ in the $\mathrm{nPM}-\mathrm{SAH}$ group with the mean age of $53.98 \pm 7.7$ years. There were no significant differences between the two groups based on age, sex, smoking history, diabetes, hypertension, anticoagulation medication history, and HH grade at admission. The nPM-SAH group was significantly associated with a higher incidence of radiological and clinical vasospasm $(p<0.05)$. Moreover, the need for external ventricular drainage (EVD) placement because of the development of hydrocephalus was significantly higher in the nPM group $(p<0.05)$. Patients with PM-SAH had better clinical outcomes than those with nPM-SAH $(p=0.037)$.

Conclusions Our results showed that patients with nonaneurysmal subarachnoid hemorrhage (NSAH) had favorable clinical outcomes. The PM group had better clinical outcomes and lower complication rates in comparison with the nPM group. Repeated digital subtraction angiography (DSA) examinations are strongly recommended for patients with nPM-SAH.
\end{abstract}

DOI https://doi.org/ $10.1055 / s-0040-1714302$ ISSN 2277-954X.
(C) 2020. Neurological Surgeons' Society of India.

This is an open access article published by Thieme under the terms of the Creative Commons Attribution-NonDerivative-NonCommercial-License, permitting copying and reproduction so long as the original work is given appropriate credit. Contents may not be used for commercial purposes, or adapted, remixed, transformed or built upon. (https://creativecommons.org/ licenses/by-nc-nd/4.0/)

Thieme Medical and Scientific Publishers Pvt. Ltd., A-12, 2nd Floor, Sector 2, Noida-201301 UP, India 


\section{Introduction}

The rupture of intracranial aneurysms is the most common cause of nontraumatic subarachnoid hemorrhage (NSAH) and is usually accompanied by a high-rate of morbidity and mortality. ${ }^{1,2}$

Despite the use of high quality four to six vessel cerebral digital subtraction angiography (DSA), the obvious source of bleeding is not detected in nearly $15 \%$ (5-30\%) of patients with NSAH. ${ }^{3,4}$

Some studies suggested a ruptured perforating artery, low-flow vascular malformation, capillary or venous source, and short segment arterial dissection as a probable source of the angiogram-negative SAH., 5 On the other hand, some authors did not agree with this hypothesis and proposed vasospasm, spontaneous thrombosis, alterations in blood flow, narrowing of the aneurysmal neck, inadequate angiographic technique, or observer error as the factors responsible for this event. ${ }^{5,7}$

Overall, nontraumatic nonaneurysmal subarachnoid hemorrhage (NNSAH) has a benign clinical course and has a generally more favorable outcome in comparison with aneurysmal SAH..$^{3,8}$

Based on the distribution pattern of the $\mathrm{SAH}$, these cases are usually divided into two subgroups, perimesencephalic (PM-SAH) and nonperimesencephalic subarachnoid hemorrhage (nPM-SAH). ${ }^{4,9}$

PM-SAH is usually associated with lower complication rates and more favorable outcomes. ${ }^{3,9}$ However, there is a controversy about the clinical outcomes and complications of patients with nPM-SAH. ${ }^{10}$ Meanwhile, recent studies recommend that the management of the nPM-SAH group should be more rigorous, keeping in mind its more severe clinical courses and outcomes. ${ }^{3,9}$

The present study aimed to investigate the risk factors, complications, and clinical outcomes of patients with NNSAH who were admitted to our center between April 2009 and April 2019.

\section{Methods}

We retrospectively evaluated 498 consecutive patients with NSAH admitted in our center between April 2009 and April 2019. SAH was diagnosed by CT and/or lumbar puncture. All patients were investigated by CTA on admission, followed by an emergent DSA examination.

There were 91 (18.27\%) SAH patients who had a negative initial CTA finding to demonstrate a culprit lesion.

Of these, initially, 13 patients had positive DSA results (two with a PM-SAH and 11 with a nPM-SAH). Finally, $78 \mathrm{SAH}$ patients with initial negative DSA findings were included in the present study.

The patients were divided into the PM-SAH ( $n=45$ patients) and the nPM-SAH groups $(n=33)$ based on the bleeding pattern on the initial CT scan. ${ }^{9,11}$

All patients underwent cranial and spinal MRI for the recognition of possible underlying causes. If a vascular lesion was suspected, additional spinal DSA was performed. Repeat
DSA evaluations were performed for 10 to 14 days following the initial DSA. Radiological vasospasm was defined as the segmental vasoconstriction of cerebral arteries, as shown by the DSA. ${ }^{3}$ The clinical vasospasm was defined as a severe headache with or without focal neurological deficits or seizures, with no evidence of rebleeding in the presence of radiological vasospasm. ${ }^{3}$ The demographic data and clinic records of all patients, including age, sex, smoking history, hypertension, diabetes, history of anticoagulant medication, Glasgow coma score (GCS), Hunt-Hess $(\mathrm{H}-\mathrm{H})$ grade, and in-hospital complications were retrospectively reviewed and compared between the two groups.

The outcomes were evaluated using the Glasgow outcome scale (GOS) at the time of discharge..$^{5,9}$ We considered GOS = 4 to 5 as a favorable outcome and GOS $=1$ to 3 as unfavorable outcomes. $^{12}$

\section{Statistical Analysis}

All statistical analyses were conducted using SPSS for Windows version 22.0 (SPSS Inc. Chicago, IL, USA). A student's $t$-test was used to compare quantitative variants. Qualitative variants were compared using the Chi-squared test or Fisher's exact test. $p<0.05$ was considered statistically significant.

\section{Results}

There were 41 men (52.56\%) and 37 women (47.43\%) with the mean age of $53.98 \pm 7.7$ years. In the present study, 45 (57.69\%) patients were in the PM-SAH group and 33 cases $(42.30 \%)$ were in the nPM-SAH group. There were no significant differences between the two groups based on age, sex, smoking history, diabetes, hypertension, anticoagulation medication history, and $\mathrm{H}-\mathrm{H}$ grades on admission (-Table 1). However, a significant difference was found between the two groups with regard to GCS score on admission ( $p=0.012$ ) (-Table 1 ).

Following the negative initial DSA, cranial MRI was performed on all patients within the first 10 days after SAH. These evaluations revealed a cavernoma as the underlying cause of bleeding in three cases (3.84\%), all of which belonged to the nPM group (-Table 2 ).

Five $(6.41 \%)$ patients were found to have an intracranial aneurysm on the second angiogram, all of which belonged to the nPM-SAH group. All five aneurysms detected in the repeat DSA investigation were anterior communicating artery aneurysms and treated with clipping or coiling.

Spinal pathologies were found in two patients $(2.56 \%)$. The cervical MRI showed two spinal arteriovenous malformations (AVMs) in the nPM-SAH group. The patients with spinal AVMs underwent endovascular treatment.

The nPM-SAH group was significantly associated with a higher incidence of radiologic and clinical vasospasm $(p<0.05)$ ( - Table 2).

Four patients (5.12\%) experienced rebleeding but no definite cause of bleeding was found even though a repeat DSA was conducted. 
Table 1 Patient characteristics

\begin{tabular}{|c|c|c|c|c|c|}
\hline & & NSAH & PM-SAH (\%) & nPM-SAH (\%) & $p$-Value \\
\hline \multicolumn{2}{|c|}{ No. of patients } & $78(100 \%)$ & $45(57.69 \%)$ & $33(42.30 \%)$ & $\mathrm{N} / \mathrm{A}$ \\
\hline \multicolumn{2}{|c|}{ Age } & $53.98 \pm 7.7$ & $53.11 \pm 8.4$ & $54.71 \pm 7.4$ & 0.433 \\
\hline \multicolumn{2}{|l|}{ Smoker (\%) } & $23(29.4 \%)$ & $15(33.3 \%)$ & $8(24.24 \%)$ & 0.721 \\
\hline \multicolumn{2}{|c|}{ Diabetes (\%) } & 17 (21.79\%) & $9(20.0 \%)$ & $8(24.24 \%)$ & 0.691 \\
\hline \multicolumn{2}{|c|}{ Hypertension (\%) } & $38(50.66 \%)$ & $21(46.66 \%)$ & 17 (51.51\%) & 0.554 \\
\hline \multicolumn{2}{|c|}{ Anticoagulant consumption (\%) } & $6(7.69 \%)$ & $4(8.88 \%)$ & $2(6.06 \%)$ & 0.912 \\
\hline \multirow[t]{3}{*}{ GCS } & Severe $3-8$ & $3(3.84 \%)$ & $0(0.00 \%)$ & $3(9.09 \%)$ & \multirow[t]{3}{*}{0.012} \\
\hline & Moderate ${ }^{9-12}$ & $10(12.8 \%)$ & $3(6.66 \%)$ & $7(21.21 \%)$ & \\
\hline & Mild $^{13-15}$ & $65(83.33 \%)$ & $42(93.33 \%)$ & 23 (69.69\%) & \\
\hline \multirow[t]{2}{*}{$\mathrm{H}$-H grade } & Good (I-II) & $71(91.02 \%)$ & $43(95.5 \%)$ & $28(84.84 \%)$ & \multirow[t]{2}{*}{0.083} \\
\hline & Poor (III-IV) & $7(8.97 \%)$ & $2(4.44 \%)$ & $5(15.15 \%)$ & \\
\hline \multirow[t]{2}{*}{$\begin{array}{l}\text { Clinical } \\
\text { outcome }\end{array}$} & $\begin{array}{l}\text { Favorable } \\
(\text { GOS }=4-5)\end{array}$ & $74(94.8 \%)$ & 45 (100.00\%) & $29(87.87 \%)$ & \multirow[t]{2}{*}{0.037} \\
\hline & $\begin{array}{l}\text { Unfavorable } \\
(\mathrm{GOS}=1-3)\end{array}$ & $4(5.12 \%)$ & $0(0.00 \%)$ & $4(12.12 \%)$ & \\
\hline
\end{tabular}

Abbreviations: GCS, Glasgow coma scale; GOS, Glasgow outcome scale; $\mathrm{H}-\mathrm{H}$ grade, Hunt-Hess grade; NSAH, nonaneurysmal subarachnoid hemorrhage; PM, perimesencephalic; nPM, nonperimesencephalic; SAH, subarachnoid hemorrhage.

Table 2 Imaging modalities for further investigation and complications

\begin{tabular}{|l|l|l|l|l|}
\hline & NSAH & PM-SAH (\%) & nPM-SAH (\%) & -Value \\
\hline Repeat DSA (positive) & $5(6.41 \%)$ & $0(0.00 \%)$ & $5(15.15 \%)$ & 0.237 \\
\hline Cranial MRI (positive) & $3(3.84 \%)$ & $0(0.00 \%)$ & $3(9.09 \%)$ & 0.67 \\
\hline Spinal MRI (positive) & $2(2.56 \%)$ & $0(0.00 \%)$ & $2(6.06 \%)$ & 0.316 \\
\hline Radiologic vasospasm & $17(21.79 \%)$ & $4(8.88 \%)$ & $13(39.39 \%)$ & 0.023 \\
\hline Clinical vasospasm & $11(14.10 \%)$ & $2(4.44 \%)$ & $9(27.27 \%)$ & 0.041 \\
\hline Early rebleeding & $4(5.12 \%)$ & $0(0.00 \%)$ & $4(12.12 \%)$ & 0.511 \\
\hline EVD placement & $7(8.97 \%)$ & $0(0.00 \%)$ & $7(21.21 \%)$ & 0.032 \\
\hline Ventriculoperitoneal shunt & $3(3.84 \%)$ & $0(0.00 \%)$ & $3(9.09 \%)$ & $13.11 \pm 7.3$ \\
\hline Mean hospital stays & $9.8 \pm 6.4$ & $8.3 \pm 6.4$ & 0.67 & 0.038 \\
\hline
\end{tabular}

Abbreviation: DSA, digital subtraction angiography; EVD, external ventricular drain; NSAH, nonaneurysmal subarachnoid hemorrhage; PM, perimesencephalic; $\mathrm{nPM}$, nonperimesencephalic; SAH, subarachnoid hemorrhage.

Hydrocephalus, which needed external ventricular drainage (EVD) placement, developed in seven patients $(8.97 \%)$, and permanent cerebrospinal fluid (CSF) diversion with ventriculoperitoneal shunts was done in three patients (3.84\%), all of which belonged to the nPM-SAH group (-Table 2).

The need for an EVD placement because of the development of hydrocephalus was significantly higher in the nPM group ( $p<0.05$ ). However, no significant difference was detected between the two groups based on the need for a permanent CSF diversion. Meanwhile, there was a significant difference between patients who developed hydrocephalus and those without hydrocephalus, based on the presence of intraventricular hemorrhage (5/7 [71.4\%] compared with 11/71 [15.49\%], [ $p<0.05])$.

The mean hospital stays of the patients with nPM-SAH (13.11 \pm 7 .3days) were significantly longer than that in the patients with PM-SAH ( $8.3 \pm 6.4$ days, $p=0.038)$.
Our results showed that patients with PMN-SAH experienced a better clinical outcome than those with nPM-SAH $(p=0.037)$ (-Table 2$)$. We had only one mortality $(1.28 \%)$ which belonged to the nPM group.

\section{Discussion}

Our results showed a better clinical outcome for patients with PM-SAH compared with those with nPM-SAH. Moreover, patients in the PM group had a lower rate of complications. It has been shown that patients with NSAH have a more favorable clinical outcome and a lower incidence of complications in comparison with those with aneurysmal SAH. ${ }^{13,14}$ Most studies reported a lower chance of complications for patients with PM-SAH. ${ }^{3,15}$ However, there is a controversy exists in relation to the clinical outcomes and complications of patients with nPM-SAH. ${ }^{10}$ Andaluz and Zuccarello reported that patients with nPM-SAH have clinical outcomes and 
complication rates similar to those with aneurysmal SAH. Furthermore, they found longer hospital and intensive care stays, worse outcomes, and higher complication rates for the nPM group compared with PM group. ${ }^{16}$ Besoglu et al reported poorer long-term clinical outcomes and less favorable quality of life scores for patients with nPM-SAH than those with PM-SAH. ${ }^{17}$ Our findings are compatible with these studies. In a retrospective study in 2017, Liang Xu et al evaluated 137 patients with NSAH. Their results showed that patients with PM-SAH had a better clinical outcome than those with nPM-SAH at 1-year follow-up. Furthermore, cases with PM-SAH had a lower rate of complications. ${ }^{9}$ In another study, Konczalla et al investigated 125 subjects with NSAH. They reported a favorable outcome for $83 \%$ of their cases. In their study, good clinical outcome was related to good admission status, absence of hydrocephalus, and younger age. ${ }^{4}$

In our study, the PMN-SAH group was significantly associated with a lower incidence of radiological and clinical vasospasm.

Akcakaya et al evaluated 81 patients with angiographic-negative SAH. They dichotomized patients into three groups as follows: PM-SAH group (40.7\%), nPM-SAH group (50.6\%), and CT-negative group (8.6\%).

Repeated DSA was positive in only two cases (2.5\%), both in the nPM group. Moreover, spinal MRI revealed the responsible lesion in all the three patients (7.3\%) belonged to the nPM group. However, cranial MRI results were negative in all of the patients.

Patients with perimesencephalic bleeding had shorter hospital stays and lower Fisher grades at the admission CT-scans. ${ }^{3}$

Because of the benign course and lower mortality and morbidity rates of NSAH, some experts do not agree with further DSA investigations for cases with initial negative angiograms. ${ }^{18,19}$ On the other hand, some studies showed that repeated DSA could reveal the culprit lesion in 2 to $21 \%$ of NSAH patients. ${ }^{20,21}$ In the present study, repeat DSA investigations were positive in five patients $(6.41 \%)$. Some studies suggested a variety of conditions, including vasospasm, thrombosis, the mass effect caused by hematoma, or technical issues such as the probable causes of initial negative angiogram. ${ }^{3,22}$

Some studies recommended cranial MRI within the first week following the SAH. ${ }^{3}$

A cranial MRI can detect angiographic negative vascular malformation-like cavernoma.,22 Andaluz and Zuccarello performed cranial MRI on 92 patients of their study group with no positive results ${ }^{16}$ Topcuoglu et al performed cranial MRI on 49 patients with NNSAH. Their results were also $100 \%$ negative.

Some studies have reported spinal lesions as a source of bleeding in approximately 1 to $3 \%$ of cases with NSAH. ${ }^{16,23}$ However, the significance value of spinal MRI as a diagnostic modality for NSAH has not been evaluated in clinical studies.
Spinal pathologies were found in two cases $(2.56 \%)$ of our patient series.

Maslehaty et al performed craniocervical MRI in all 179 patients with NSAH and reported negative results in all of the patients. ${ }^{24}$

Germans et al detected a 9\% incidence of spinal pathologies in their patients with nPM-SAH. ${ }^{25}$ However, they performed whole spine MRI in 51 patients with PM-SAH and reported $100 \%$ negative results. ${ }^{26}$

\section{Limitations}

The present study has several limitations. This is a retrospective study and some confounding variables may have not been measured and collected due to the retrospective nature of this study. Furthermore, the single-center study could have limited the generalizability of our findings despite the good outcome. Further investigations with long-term functional, psychological, and social outcomes would be of great interest.

\section{Conclusions}

Our results showed that patients with NSAH had favorable clinical outcomes. The PM group had better clinical outcomes and lower complication rates in comparison with the nPM group. Repeated DSA examinations are strongly recommended for patients with nPM-SAH.

\section{Note}

All data are available from the corresponding author upon reasonable request.

\section{Ethical Approval}

The study received ethical approval by the Kermanshah University of Medical Science Ethics Committee.

\section{Funding \\ None.}

\section{Authors' Contributions}

E.A. and S.R.B. had the idea for this study. E.A., P.A., A.K., and A.A. participated in outlining the concept and design. P.A. and S.H.R. did the data acquisition. E.A. and S.H.R. did the statistical analysis and wrote the first draft of the manuscript. E.A., S.R.B., P.A., and A.K. revised the final manuscript. All authors have read and approved the manuscript.

\section{Conflict of Interest}

None declared.

\section{Acknowledgments}

The authors appreciate the Clinical Research Development Center of Taleghani and Imam Ali Hospitals for their valuable suggestions. 


\section{References}

1 Khan AA, Smith JD, Kirkman MA, et al. Angiogram negative subarachnoid haemorrhage: outcomes and the role of repeat angiography. Clin Neurol Neurosurg 2013;115(8):1470-1475

2 Bruder N. [Non-aneurysmal subarachnoid haemorrhage]. Ann Fr Anesth Reanim 2007;26(11):954-958

3 Akcakaya MO, Aydoseli A, Aras Y, et al. Clinical course of non-traumatic non-aneurysmal subarachnoid hemorrhage: a single institution experience over 10 years and review of the contemporary literature. Turk Neurosurg 2017;27(5):732-742

4 Konczalla J, Platz J, Schuss P, Vatter H, Seifert V, Güresir E. Nonaneurysmal non-traumatic subarachnoid hemorrhage: patient characteristics, clinical outcome and prognostic factors based on a single-center experience in 125 patients. BMC Neurol 2014; $14: 140$

5 Pyysalo LM, Niskakangas TT, Keski-Nisula LH, Kähärä VJ, Öhman JE. Long term outcome after subarachnoid haemorrhage of unknown aetiology. J Neurol Neurosurg Psychiatry 2011;82(11):1264-1266

6 van der Schaaf IC, Velthuis BK, Gouw A, Rinkel GJ. Venous drainage in perimesencephalic hemorrhage. Stroke 2004;35(7): 1614-1618

7 McMahon J, Dorsch N. Subarachnoid haemorrhage of unknown aetiology: what next. ? Crit Rev Neurosurg 1999;9(3):147-155

8 Jabbarli R, Reinhard M, Roelz R, et al. Outcome prediction after non-aneurysmal non-traumatic subarachnoid hemorrhage. Curr Neurovasc Res 2015;12(3):269-276

9 Xu L, Fang Y, Shi X, et al. Management of spontaneous subarachnoid hemorrhage patients with negative initial digital subtraction angiogram findings: conservative or aggressive? BioMed Res Int 2017;2017:2486859

10 Reynolds MR, Blackburn SL, Zipfel GJ. Recurrent idiopathic perimesencephalic subarachnoid hemorrhage. J Neurosurg 2011;115(3):612-616

11 Wallace AN, Vyhmeister R, Dines JN, et al. Evaluation of an anatomic definition of non-aneurysmal perimesencephalic subarachnhoid hemorrhage. J Neurointerv Surg 2016;8(4):378-385

12 Teasdale GM, Pettigrew LE, Wilson JT, Murray G, Jennett B. Analyzing outcome of treatment of severe head injury: a review and update on advancing the use of the Glasgow Outcome Scale. J Neurotrauma 1998;15(8):587-597

13 Kong Y, Zhang JH, Qin X. Perimesencephalic subarachnoid hemorrhage: risk factors, clinical presentations, and outcome. In Early Brain Injury or Cerebral Vasospasm 2011 (pp. 197-201). Vienna: Springer

$14 \mathrm{Du} \mathrm{CP}, \mathrm{Ke} \mathrm{KF}$. [The clinical analysis of nonaneurysmal subarachnoid hemorrhage]. Zhonghua Nei Ke Za Zhi 2011;50(5): 408-410
15 Kapadia A, Schweizer TA, Spears J, Cusimano M, Macdonald RL. Nonaneurysmal perimesencephalic subarachnoid hemorrhage: diagnosis, pathophysiology, clinical characteristics, and long-term outcome. World Neurosurg 2014;82(6):1131-1143

16 Andaluz N, Zuccarello M. Yield of further diagnostic work-up of cryptogenic subarachnoid hemorrhage based on bleeding patterns on computed tomographic scans. Neurosurgery 2008;62(5):1040-1046, discussion 1047

17 Beseoglu K, Pannes S, Steiger HJ, Hänggi D. Long-term outcome and quality of life after nonaneurysmal subarachnoid hemorrhage. Acta Neurochir (Wien) 2010;152(3):409-416

18 Ruigrok YM, Rinkel GJ, Buskens E. Velthuis BK, van Gijn J. Perimesencephalic hemorrhage and CT angiography: A decision analysis. Stroke 2000;31(12):2976-2983

19 Velthuis BK, Rinkel GJ, Ramos LM. Witkamp TD, van Leeuwen MS. Perimesencephalic hemorrhage. Exclusion of vertebrobasilar aneurysms with CT angiography. Stroke 1999;30(5): 1103-1109

20 Whiting J, Reavey-Cantwell J, Velat G, et al. Clinical course of nontraumatic, nonaneurysmal subarachnoid hemorrhage: a single-institution experience. Neurosurg Focus 2009;26(5):E21

21 Boswell S, Thorell W, Gogela S, Lyden E, Surdell D. Angiogramnegative subarachnoid hemorrhage: outcomes data and review of the literature. J Stroke Cerebrovasc Dis 2013;22(6):750-757

22 Topcuoglu MA, Ogilvy CS, Carter BS, Buonanno FS, Koroshetz WJ, Singhal AB. Subarachnoid hemorrhage without evident cause on initial angiography studies: diagnostic yield of subsequent angiography and other neuroimaging tests. J Neurosurg 2003;98(6):1235-1240

23 Van Calenbergh F, Plets C, Goffin J, Velghe L. Nonaneurysmal subarachnoid hemorrhage: prevalence of perimesencephalic hemorrhage in a consecutive series. Surg Neurol 1993;39(4):320-323

24 Maslehaty H, Petridis AK, Barth H, Mehdorn HM. Diagnostic value of magnetic resonance imaging in perimesencephalic and nonperimesencephalic subarachnoid hemorrhage of unknown origin. J Neurosurg 2011;114(4):1003-1007

25 Germans MR, Coert BA, Majoie CB. van den Berg R, Verbaan $D$, Vandertop WP. Spinal axis imaging in non-aneurysmal subarachnoid hemorrhage: a prospective cohort study. J Neurol 2014;261(11):2199-2203

26 Germans MR, Coert BA, Majoie CB, et al. Yield of spinal imaging in nonaneurysmal, nonperimesencephalic subarachnoid hemorrhage. Neurology 2015;84(13):1337-1340 